\title{
An Unusual Cause of Urinary Pseudoincontinence: Two Rare Cases of Labial Fusion in Adolescent and Postmenopausal Women
}

\author{
Anuradha Murugesan ${ }^{1}$, Yegu Palaniappan ${ }^{2}$, Thendral Kalaimani ${ }^{3}$
}

\begin{abstract}
Labial adhesions are usually a pediatric condition and are asymptomatic. They are identified by parents or on routine examination. They can also occur in postmenopausal age group albeit uncommon due to hypoestrogenism causing senile vaginitis. Here, we have two cases of labial fusion in different age groups, 17-years-old and 65-year-old females presenting with urinary symptoms. The release of labial fusion was done under general anesthesia in both the patients. The labial adhesions are usually a pediatric condition and are asymptomatic. Although one needs to have high index of suspicion to diagnose this rare condition, as the patient often presents with common gynecological symptoms of difficult menstruation and urinary dribbling. It can also occur in postmenopausal women though uncommon, one needs to have high index of suspicion due to hypoestrogenism.

Keywords: Incontinence, Labial fusion, Postmenopausal, Postpubertal.

Journal of South Asian Federation of Obstetrics and Gynaecology (2020): 10.5005/jp-journals-10006-1789
\end{abstract}

\section{INTRODUCTION}

Labial adhesions are usually a pediatric condition and are asymptomatic. They are identified by parents or on routine examination. They can also occur in postmenopausal age group albeit uncommon due to hypoestrogenism causing senile vaginitis. ${ }^{1,2}$ It is even more rarely seen in reproduction age group. ${ }^{3}$ Clinical presentation can be with urinary or vulvar symptoms. The proposed factors for labial adhesion include hypoestrogenism, chronic irritation, infections of the vulva, and recurrent urinary tract infections. ${ }^{3}$ Herein, we describe two cases of labial adhesion in the extremes of age group presenting with similar symptoms of dribbling of urine.

\section{Case Descriptions}

\section{Case 1}

A 17-year-old adolescent girl sexually inactive presented to our Outpatient Department of Gynecology in January 2017 with chief complaints of thin stream of urine and dribbling of urine on standing for the past 6 months. She attained menarche at the age of 13 and had regular menstrual cycles with the history of dysmenorrhea. She had recurrent episodes of UTI and genital irritation. There was no history of fall or sexual abuse.

A detailed history and physical examination revealed normal secondary sexual characters with fused labia minora with a pinhole opening. Urethra and vagina could not be visualized. The anus and perineum were normal (Fig. 1).

She had similar complaints 1 year after menarche and diagnosed as labial fusion at an outside hospital and underwent release of adhesions under general anesthesia. Cystoscopy and vaginoscopy were performed, and the findings were normal. Also, she was treated with a course of antibiotics. We began her work-up in our hospital, with a vaginal swab culture and sensitivity, done under aseptic precautions through the small pinhole opening.
${ }^{1-3}$ Department of Obstetrics and Gynaecology, SRM Medical College and Research Centre, Chennai, Tamil Nadu, India

Corresponding Author: Anuradha Murugesan, Department of Obstetrics and Gynaecology, SRM Medical College and Research Centre, Chennai, Tamil Nadu, India, Phone: +91 9042038136, e-mail: anuthanigai@yahoo.com

How to cite this article: Murugesan A, Palaniappan Y, Kalaimani T. An Unusual Cause of Urinary Pseudoincontinence: Two Rare Cases of Labial Fusion in Adolescent and Postmenopausal Women. J South Asian Feder Obst Gynae 2020;12(3):196-198.

Source of support: Nil

Conflict of interest: None

Routine urine analysis, urine culture and sensitivity, and renal function tests were done showing no abnormalities. TB Gold

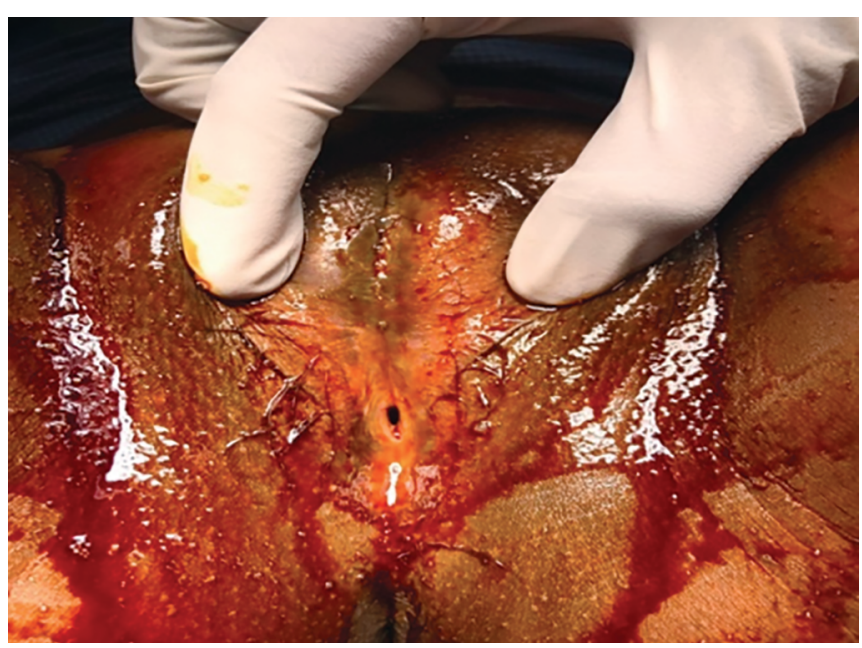

Fig. 1: Fused labia (postpubertal girl)

(0) The Author(s). 2020 Open Access This article is distributed under the terms of the Creative Commons Attribution 4.0 International License (https://creativecommons. org/licenses/by-nc/4.0/), which permits unrestricted use, distribution, and non-commercial reproduction in any medium, provided you give appropriate credit to the original author(s) and the source, provide a link to the Creative Commons license, and indicate if changes were made. The Creative Commons Public Domain Dedication waiver (http://creativecommons.org/publicdomain/zero/1.0/) applies to the data made available in this article, unless otherwise stated. 


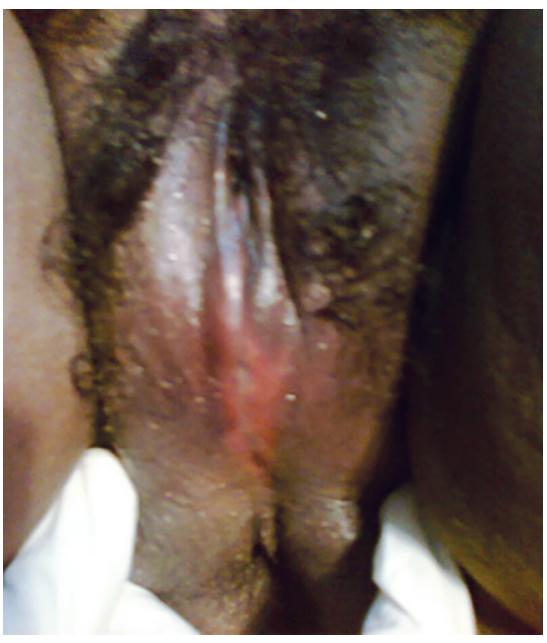

Fig. 2: Labial fusion (postmenopausal)

test was done to rule out genital tuberculosis and was negative. Ultrasound KUB and pelvis were normal, complete hemogram was within normal limits, and her serology for HIV, syphilis, and hepatitis B were found to be negative.

Intraoperative findings-both labia minora appear fused. A pinhole opening was visible near the fourchette. The release of labial adhesions was done under general anesthesia. Cervix, vagina, and urethra were found to be normal. Skin edges were everted and sutured with 1-0 catgut. Patient was given estrogen cream for daily application for 3 months. Patient is on follow-up till now and there is no recurrence.

\section{Case 2}

A 65-year-old postmenopausal women P3L3 presented to our Outpatient Department of Gynecology with the complaints of abdominal pain and dribbling of urine. She attained menopause 10 years back. She had history of white discharge and pruritus vulva. On examination, labia minora were found to be fused similar to the above case. Urethra and vagina could not be seen, and the urine was dribbling through pinhole os (Fig. 2). There were no similar complaints in the past.

Under short GA, both labia minora were separated and there was a pool of urine in the vagina. Urethra was normal and about $1,000 \mathrm{~mL}$ of urine was drained after catheterization, and the cut edges were everted and sutured with 1-0 catgut. Patient was prescribed with estrogen cream for 3 months, and the patient did not turn up for the follow-up visits.

\section{Discussion}

Labial fusion is defined as the partial or complete fusion of the labia minora causing partial or complete occlusion of the vaginal and/ or urethral opening. It can be congenital or acquired. Associated ambiguous genitalia, vaginal agenesis, hypoplastic kidney and microperforate hymen can be seen in congenital labial fusion. Labial fusion is often an acquired condition typically seen in prepubertal girls, usually below 6 years of age. ${ }^{4}$ It is uncommonly seen in postmenopausal woman and even more rarely in the reproduction age group. Worldwide incidence is not known. It is reported to occur in $1-2 \%$ of females aged 3 months to 6 years in the United States. ${ }^{5}$

Estrogen deficiency plays a major role in the development of labial adhesion, thereby explaining its high incidence rate in the prepubertal females and it is the cause in the postmenopausal age. It can present with vulvar symptoms such as burning, itching, pain, dyspareunia, and discomfort during menstruation, vaginal discharge, recurrent urinary tract infections, and dribbling of urine.

Our case is unique for two reasons: presentation in the postpubertal age and recurrence after surgery. There are only few case reports of labial fusion occurring in the postpubertal reproductive woman. ${ }^{3,6,7}$ The reported causes in this age group include poor hygiene, dermatitis, nonspecific or candidal vaginitis, herpes simplex, trauma due to fall, female circumcision, and lichen sclerosis. There have also been reports of LA following vaginal delivery and spontaneous closure during pregnancy. ${ }^{8}$ Patients often present with urinary dribbling and/or menstrual difficulties.

Diagnosis is made on physical examination which shows the fused labia minora with or without the involvement of labia majora. The typical appearance is a flat appearing genitalia and a midline vertical translucent membrane which can be thin and fragile or thick and dense. ${ }^{9}$ Differential diagnosis includes vaginal atresia, Gartner's duct cyst, ambiguous genitalia and imperforate hymen in children, and mass occupying conditions such as skin tags, polyp, urethrocele, prolapse or diverticulum of urethra needs in older females. ${ }^{2,4,7,10,11}$ It needs to be distinguished from congenital deformities which often give the appearance that the opening of the vagina has closed off, by the identification of a midline raphe seen in the labial fusion. ${ }^{9}$

In doubtful cases, a detailed examination under anesthesia, cystoscopy, voiding cystourethrography can be helpful. A complete work-up should include ultrasound abdomen and pelvis to rule out associated genitourinary anomalies, uterine anomalies, hydro, or hematometrocolpos in severe cases. Urine culture and high vaginal swabs are recommended before definitive management.

Various treatment options include topical estrogen, topical betamethasone, manual, and surgical separation. The choice of treatment depends on the age group and the type of adhesions. In the postpubertal group, often manual or surgical separation is required as they are often thick and dense. Conjugated estrogen cream or estradiol vaginal cream $(0.01 \%)$ is applied to the adhesions 1-2 times daily for several weeks until the adhesion resolves. Alternatively, topical $0.05 \%$ betamethasone for $4-6$ weeks can be considered for one to three courses twice everyday. ${ }^{12,13}$ Manual separation can be tried in thin adhesions not responding to medical management. But traumatic lysis should be avoided as it can lead to recurrence with the development of more dense adhesions. Surgical separation is reserved for thick dense adhesions, thin adhesions not responding to other treatments and recurrent cases. ${ }^{14,15}$ Both patients had thick adhesions. Hence, surgical separation was performed with postoperative application of topical estrogen and antibiotics to promote healing and prevent recurrence. Surgical technique includes division of adhesions by gentle traction or running a sound along the fusion line. ${ }^{10}$

Recurrent labial fusion is a difficult situation both for the patient and for the treating gynecologist. It is not uncommon reported to occur in $11-14 \%$. It can be seen after treatment in all age groups and after all forms of treatment. In a study involving 48 cases with recurrence, it was found that $83 \%$ of recurrences were following topical estrogen use alone, and in $14 \%$ patients, the recurrence was following manual separation with or without topical estrogen. ${ }^{16}$ Apart from the routine surgical separation, alternative/additional surgical techniques include the use of amniotic membrane, 
rotational skin flaps, split thickness skin grafts and suturing pieces of saline infusion tubes to the raw area. ${ }^{12,17,18}$

\section{CONCLUSION}

Although uncommon, labial fusion can occur in the postpubertal females, one needs to have high index of suspicion to diagnose this rare condition as the patient often presents with common gynecological symptoms of difficult menstruation and urinary dribbling. This case illustrates the value of gynecological examination for the evaluation of voiding symptoms as otherwise disorders such as labial adhesion, meatal stenosis, and ambiguous genitalia may not be recognized and carries forward to the reproductive age. Physical examination is often adequate for the diagnosis and can be complimented by cystoscopy and ultrasound examinations. Apart from surgical expertise, identification of causative factor is important to prevent recurrent labial fusion.

\section{References}

1. Julia J, Yacoub M, Levy G. Labial fusion causing urinary incontinence in a postmenopausal female: a case report. Int Urogynecol J Pelvic Floor Dysfunct 2003;14(5):360-361. DOI: 10.1007/s00192-0031067-y.

2. Aizawa $\mathrm{T}$, Ishibashi $\mathrm{K}$, Oyama $\mathrm{H}$, et al. A case of labial adhesions in an elderly woman. Hinyokika Kiyo 1998;44:129-132.

3. Erdogdu E, Demirel C, Tahaoglu AE, et al. Labial fusion: a rare cause of urinary retention in reproductive age woman and review of literature. Turk J Urol 2017;43(1):98-101. DOI: 10.5152/tud.2017.58897.

4. Kumar RK, Sonika A, Charu C, et al. Labial adhesions in pubertal girls. Arch Gynecol Obstet 2006;273(4):243-245. DOI: 10.1007/s00404-0050060-8.

5. Leung AK, Robson WL, Tay-Uyboco J. The incidence of labial fusion in children. J Paediatr Child Health 1993;29(3):235-236. DOI: 10.1111/ j.1440-1754.1993.tb00495.x.
6. Kucuk M, Halil S, Ocer F, et al. Labial fusion first diagnosed during pregnancy with voiding difficulty and its management: a case report. Clin Exp Obstet Gynecol 2011;38:94-95.

7. Topcuoglu MA, Koc O, Duran B, et al. Labial fusion causing acute urinary retention in a young adult: a case report. Aust N Z J Obstet Gynaecol 2009;49(1):115-116. DOI: 10.1111/j.1479-828X.2008.00943.x.

8. Lin $\mathrm{YH}$, Hwang JL, Huang LW, et al. Amniotic membrane grafting to treat refractory labial adhesions postpartum. A case report. J Reprod Med 2002;47:235-237.

9. Js E. Congenital Anomalies of the Genitalia. 6th ed., 1992.

10. Dirim A, Hasirci E. Labial fusion causing urinary incontinence and recurrent urinary tract infection in a postmenopausal female: a case report. Int Urogynecol J 2011;22(1):119-120. DOI: 10.1007/s00192-0101205-2.

11. Starr NB. Labial adhesions in childhood. J Pediatr Health Care 1996;10(1):26-27. DOI: 10.1016/S0891-5245(96)90070-X.

12. Mayoglou L, Dulabon L, Martin-Alguacil N, et al. Success of treatment modalities for labial fusion: a retrospective evaluation of topical and surgical treatments. J Pediatr Adolesc Gynecol 2009;22(4):247-250. DOI: 10.1016/j.jpag.2008.09.003.

13. Myers JB, Sorensen CM, Wisner BP, et al. Betamethasone cream for the treatment of pre-pubertal labial adhesions. J Pediatr Adolesc Gynecol 2006;19(6):407-411. DOI: 10.1016/j.jpag.2006.09.005.

14. Nevarez Bernal RA, Meraz Avila D. Fusion of the labia minora as a cause of urinary incontinence in a postmenopausal woman. a case report and literature review. Ginecol Obstet Mex 2009;77:287-290.

15. Palla $L$, De Angelis $B$, Lucarini $L$, et al. A case of labial fusion and urinary pseudo-incontinence in an elderly woman. A surgical treatment and a review. Eur Rev Med Pharmacol Sci 2010;14:491-493.

16. Kumetz LM, Quint EH, Fisseha S, et al. Estrogen treatment success in recurrent and persistent labial agglutination. J Pediatr Adolesc Gynecol 2006;19(6):381-384. DOI: 10.1016/j.jpag.2006.09.008.

17. Johnson N, Lilford RJ, Sharpe D. A new surgical technique to treat refractory labial fusion in the elderly. Am J Obstet Gynecol 1989;161(2):289-290. DOI: 10.1016/0002-9378(89)90500-0.

18. Silva $D$, Jayalath GK, Ranaweera AK, et al. A new method of surgical treatment for recurrent labial adhesions in a pre-pubertal girl. Ceylon Med J 2012;57(4):168-169. DOI: 10.4038/cmj.v57i4.5086. 